\title{
Hyperfine-assisted decoherence of a phosphorus nuclear-spin qubit in silicon
}

\author{
Bence Hetényi, ${ }^{1,2, *}$ Péter Boross ${ }^{3}$ and András Pályi ${ }^{4}{ }^{\dagger}$ \\ ${ }^{1}$ Institute of Physics, Eötvös University, 1518 Budapest, Hungary \\ ${ }^{2}$ Department of Physics, University of Basel, Klingelbergstrasse 82, CH-4056 Basel, Switzerland \\ ${ }^{3}$ Institute for Solid State Physics and Optics, Wigner Research Centre for Physics, \\ Hungarian Academy of Sciences, H-1525 Budapest P.O. Box 49, Hungary \\ ${ }^{4}$ Department of Theoretical Physics and MTA-BME Exotic Quantum Phases Research Group, \\ Budapest University of Technology and Economics, 1111 Budapest, Hungary
}

\begin{abstract}
The nuclear spin of a phosphorus atom in silicon has been used as a quantum bit in various quantum-information experiments. It has been proposed that this nuclear-spin qubit can be efficiently controlled by an ac electric field, when embedded in a two-electron dot-donor setup subject to intrinsic or artificial spin-orbit interaction. Exposing the qubit to control electric fields in that setup exposes it to electric noise as well. In this work, we describe the effect of electric noise mechanisms, such as phonons and $1 / f$ charge noise, and estimate the corresponding decoherence time scales of the nuclear-spin qubit. We identify a promising parameter range where the electrical single-qubit operations are at least an order of magnitude faster then the decoherence. In this regime, decoherence is dominated by dephasing due to $1 / f$ charge noise. Our results facilitate the optimized design of nanostructures to demonstrate electrically driven nuclear spin resonance.
\end{abstract}

\section{INTRODUCTION}

The nuclear spin of a phosphorus (P) atom in silicon $(\mathrm{Si})$ is a highly coherent two-level system ${ }^{1-5}$, and has been used as a qubit in several quantum-information experiments $^{6-10}$. Single-qubit control of such a nuclear spin have been demonstrated using ac magnetic fields, in the spirit of nuclear magnetic resonance ${ }^{6}$; initialization and readout can be performed using the donor electron $\operatorname{spin}^{6,11,12}$.

A recent work ${ }^{13}$ proposes an engineered nanostructure, in which the $\mathrm{P}$ nuclear-spin qubit could be efficiently controlled by an ac electric field, offering several practical advantages ${ }^{13-18}$, in comparison with the traditionally used ac magnetic field. The proposed setup is a dot-donor structure with two bound electrons ${ }^{19-22}$, and the interaction between the control electric field and the nuclear spin is mediated by the interplay of hyperfine interaction and (intrinsic or artificial) spin-orbit interaction. In short, the ac electric field makes the spin of the electrons time dependent, and hyperfine interaction translates the time-dependent electronic spin to a timedependent Knight field, felt by the nuclear spin due to the hyperfine interaction. Hence, the system of the two electrons functions as a transducer, converting the ac electric field to an effective ac magnetic field for the nucleus.

The above mechanism is useful for electrical control, but it also exposes the nuclear-spin qubit to decoherence due to electric fluctuations. In this work, we describe two electrical noise mechanisms, phonons and $1 / f$ charge noise, in the setup described above. Our goal is to estimate the corresponding decoherence time scales of the nuclear-spin qubit. We identify a promising parameter range where the electrical single-qubit operations are at least an order of magnitude faster than the decoherence, and the latter is dominated by dephasing due to $1 / f$ charge noise. Our study complements earlier theory works where the decoherence of electron-spin and flip-flop qubits in the dot-donor system were described ${ }^{14,23,24}$.

The rest of the paper is organized as follows. In Section II, we introduce the model of the dot-donor setup, and describe dephasing of the nuclear-spin qubit due to $1 / f$ charge noise, which is the dominant information-loss mechanism in the considered range of parameters. Even though having two bound electrons in the dot-donor system has definite advantages over having only a single electron, we also discuss the latter case, for completeness and because of its conceptual simplicity. In Section III, we analyse qubit relaxation processes and leakage from the qubit subspace due to $1 / f$ charge noise. In Section IV, we describe how phonons contribute to relaxation and leakage. Estimates of time scales for the various setups and mechanisms are collected in Table I. A few remarks are made in Section V, and conclusions are drawn in Section VI.

\section{NUCLEAR-SPIN DEPHASING DUE TO $1 / f$ CHARGE NOISE}

In this section, we introduce the P:Si nuclearspin qubit and the special dot-donor setup that enables its electrical control. We discuss two different arrangements: ${ }^{13}$. Single-electron setup (1e): A single electron is confined in the dot-donor system. This brings the advantage of conceptual simplicity, but the quality of electric control suffers strongly from electrical noise. Two-electron setup (2e): Two electrons are confined in the system, allowing for efficient electrical control even in the presence of realistic charge noise. Our main goal then is to describe the dephasing of the nuclear-spin qubit due to $1 / f$ charge noise in both setups, to calculate the dephasing time $T_{2}^{*}$, and to identify a parameter range where the dephasing time is longer than the time scale of single-qubit operations. This is indeed possible for the 2e setup, as revealed by the last line of Table I: the esti- 
mated dephasing rate for that particular working point is $\Gamma_{2}^{*} \equiv 1 / T_{2}^{*}=2.97 \mathrm{kHz}$, whereas the Rabi frequency characterizing the operation time scale is $f_{\text {Rabi }} \approx 53 \mathrm{kHz}$.

\section{A. Nuclear-spin qubit with a single donor electron}

The setup is shown in Fig. 1. A P atom (red sphere) is embedded in a Si crystal, at distance $d$ from the interface with an insulating barrier (e.g. $\mathrm{SiO}_{2}$ ). The barrier separates the bulk $\mathrm{Si}$ from the gate electrode, the latter being used to control the position of the donor electron. An ac component of the voltage on the gate electrode can be used to electrically drive the nuclear-spin qubit; note, however, that in this work we consider only the non-driven case, when the gate voltage is dc.

At low temperature and zero gate voltage, there is a single electron bound to the donor nucleus. A finite dc gate voltage creates an electric field $\boldsymbol{E}(\boldsymbol{r})$ in its vicinity. When this electric field is pulling the electron toward the gate strongly enough, then the electron is removed from the donor and sticks to the interface with the barrier, where it is trapped in a quantum-dot-like confinement potential created by the gate electrode. Under certain conditions $^{14}$, there is a finite gate-voltage value which ensures that half of the electronic wave function is localized on the donor, and the other half is at the interface; this situation is depicted in Fig. 1a, where the gray cloud corresponds to this 'split' wave function resembling a bonding state in a diatomic molecule. We refer to this setting as the ionization point ${ }^{14}$.

If the gate voltage is tuned to the vicinity of the ionization point, then the 'orbital' or 'charge' degree of freedom of the electron can be described using the two localized orbitals $|i\rangle$ and $|d\rangle$; the former is the one localized at the interface, the latter is the one localized on the donor. Then, the $2 \times 2$ effective Hamiltonian that describes the charge in the vicinity of the ionization point can be written as $^{14}$

$$
H_{\mathrm{ch}}=\frac{U}{2} \sigma_{z}+\frac{V_{t}}{2} \sigma_{x}
$$

where $U$ is the on-site energy difference between the interface and donor orbitals (controlled by the gate voltage), $V_{t}$ is the tunnelling amplitude, and the Pauli matrices are defined in the basis above, e.g. as $\sigma_{z}=|i\rangle\langle i|-| d\rangle\langle d|$. We denote the eigenstates of $H_{\mathrm{ch}}$ as $|a\rangle$ and $|b\rangle$, as a reference to the anti-bonding (higher-energy) and the bonding (lower-energy) state. Note that a low-energy excited orbital, i.e., the valley pair of $|i\rangle$, is available at the interface ${ }^{14,21,24}$, with an excitation energy varying from a few tens to a few hundreds of microelectronvolts. We disregard this state in our minimal model, assuming that its excitation energy is much larger than the tunnel coupling.

The two-dimensional minimal model for the electron charge, introduced above, has to be extended with the electronic spin and nuclear spin degrees of freedom, yielding an $8 \times 8$ Hamiltonian

$$
H=H_{\mathrm{ch}}+H_{B, \mathrm{e}}+H_{B, \mathrm{n}}+H_{\mathrm{hf}}+H_{\mu, \mathrm{e}}+H_{\mu, \mathrm{n}} .
$$

This incorporates the effects of the homogeneous static magnetic field $\left(H_{B}\right)$, hyperfine interaction between the electronic and nuclear spin $\left(H_{\mathrm{hf}}\right)$, and intrinsic or artificial spin-orbit interaction $\left(H_{\mu}\right)$. In this work, we consider artificial spin-orbit interaction and neglect the intrinsic mechanism (see Section $\mathrm{V}$ for a discussion). More precisely, we assume the presence of an inhomogeneous magnetic field along $\mathrm{x}, B_{x}=\beta y$ (see Fig. 1a), where the origin of the $y$ axis is chosen halfway between the charge centers of the $|i\rangle$ and $|d\rangle$ orbitals. Then, the Hamiltonian terms read

$$
\begin{aligned}
H_{B, \mathrm{e}} & =h \gamma_{\mathrm{e}} B S_{z} \\
H_{B, \mathrm{n}} & =-h \gamma_{\mathrm{n}} B I_{z} \\
H_{\mathrm{hf}} & =A n_{\mathrm{d}} \boldsymbol{S} \cdot \boldsymbol{I} \\
H_{\mu, \mathrm{e}} & =h \gamma_{\mathrm{e}} \frac{\beta d}{2} \sigma_{z} S_{x} \\
H_{\mu, \mathrm{n}} & =h \gamma_{\mathrm{n}} \frac{\beta d}{2} I_{x},
\end{aligned}
$$

where $A / h=117 \mathrm{MHz}$ is the hyperfine coupling strength, while $\gamma_{\mathrm{e}}=27.97 \mathrm{GHz} / \mathrm{T}$ and $\gamma_{\mathrm{n}}=17.23 \mathrm{MHz} / \mathrm{T}$ are the electron and nuclear gyromagnetic factors. Furthermore, $\boldsymbol{B}=(0,0, B)$ is the homogeneous magnetic field, the operator $n_{\mathrm{d}}=\left(1-\sigma_{z}\right) / 2$ is the occupation number of the donor site, $\boldsymbol{S}=\left(S_{x}, S_{y}, S_{z}\right)$ is the spin of the donor electron, and $\boldsymbol{I}=\left(I_{x}, I_{y}, I_{z}\right)$ is the nuclear spin of the $\mathrm{P}$ atom. We will denote the eigenstates of $S_{z}\left(I_{z}\right)$ as $|\uparrow\rangle$ and $|\downarrow\rangle(|\Uparrow\rangle$ and $|\Downarrow\rangle)$.

Below, we will obtain analytical results for various physical quantities using perturbation theory. In those calculations, the unperturbed Hamiltonian is chosen as $H_{0}=H_{\mathrm{ch}}+H_{B, \mathrm{e}}+H_{B, \mathrm{n}}+H_{\mathrm{hf}, \mathrm{sec}}$, whereas the perturbation is $H_{1}=H-H_{0}$. Above, $H_{\mathrm{hf}, \mathrm{sec}}=A n_{\mathrm{d}} S_{z} I_{z}$ is the 'secular' or 'diagonal' part of the hyperfine interaction. We call the eigenstates of $H_{0}$ unperturbed states. These are product states formed from the previously defined charge and spin states, and therefore we denote them as, e.g., $|b \downarrow \Uparrow\rangle_{0}$. As long as the perturbation matrix elements between the unperturbed states are weak, the eigenstates of $H$ can be labelled with the same labels, such as $|b \downarrow \uparrow\rangle$.

For a particular parameter set studied in Ref. 13, the energy eigenstates of $H$ are shown as the function of the on-site energy difference $U$ in Fig. 1b. The basis states of the nuclear-spin qubit are those with the two lowest energies $\epsilon_{g}$ and $\epsilon_{e}$, highlighted by the zoom-in in Fig. 1b, denoted as $|g\rangle_{1 e} \equiv|b \downarrow \Uparrow\rangle$ and $|e\rangle_{1 e} \equiv|b \downarrow \downarrow\rangle$.

\section{B. Nuclear-spin dephasing due to $1 / \mathrm{f}$ charge noise}

Our goal here is to focus on the nuclear-spin qubit, and describe the information-loss mechanisms arising from its interaction with electrical fluctuations. Recent 

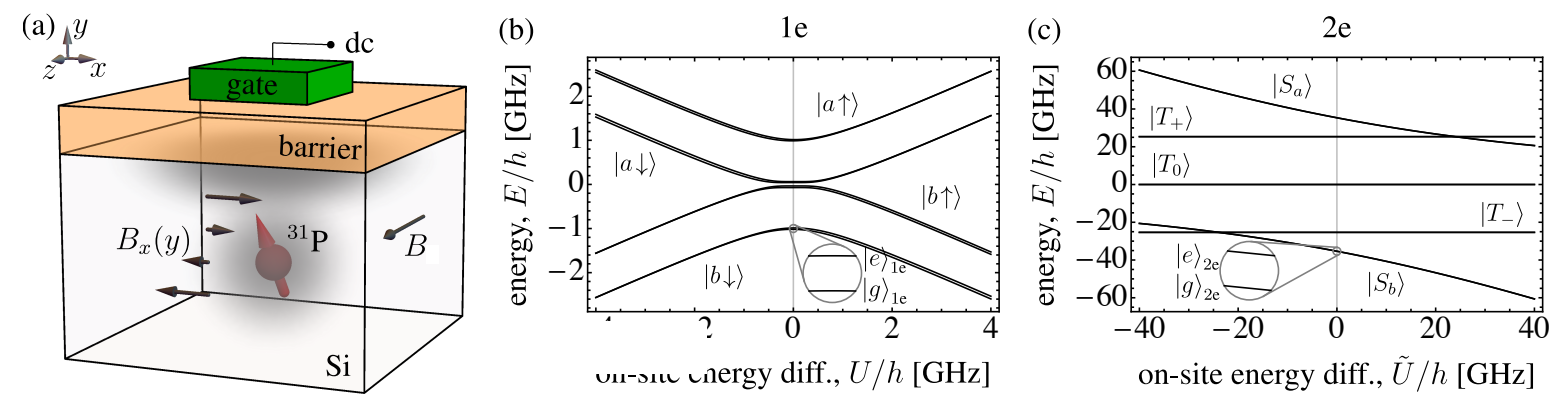

FIG. 1. Dot-donor setup and the energy spectra of the coupled electron-nuclear system. (a) Dot-donor setup. The dc gate voltage is used to balance the donor electron (or electrons) on a bonding orbital (gray cloud) of the artificial molecule formed by the dot-donor system. Magnetic field (denoted by black arrows) has a homogeneous part $B$ along the $z$ direction and an inhomogeneous $B_{x}(y)$ along $x$. The inhomogeneous magnetic field can be replaced by a sufficiently strong spin-orbit interaction. Red arrow represents the nuclear spin. (b) Energy spectrum of the coupled electron-nuclear system in the single-electron setup. Homogeneous magnetic field: $B=35.7 \mathrm{mT}$, tunnelling amplitude: $V_{t} / h=1 \mathrm{GHz}$. (c) Energy spectrum in the two-electron setup. Magnetic field: $B=906.5 \mathrm{mT}$, tunneling amplitude: $V_{t} / h=50 \mathrm{GHz}$. Zoom-ins in (b) and (c) show the two basis states of the nuclear-spin qubit.

\begin{tabular}{|c|c|c|c|c|c|c|c|c|c|c|c|c|c|}
\hline \multirow{2}{*}{\multicolumn{2}{|c|}{\begin{tabular}{|c|} 
Dephasing $[\mathrm{Hz}]$ \\
$\Gamma_{2}^{*}$
\end{tabular}}} & \multicolumn{4}{|c|}{ Relaxation $[\mathrm{Hz}]$} & \multicolumn{4}{|c|}{ Leakage $[\mathrm{Hz}]$} & \multirow[b]{2}{*}{$B[\mathrm{~T}]$} & \multirow[b]{2}{*}{$V_{t} / h$} & \multirow[b]{2}{*}{$f_{\text {Rabi }}$} & \multirow[b]{2}{*}{$f_{\mathrm{L}}$} \\
\hline & & $\Gamma_{\mathrm{R}, \mathrm{p}}^{e}$ & $\Gamma_{\mathrm{R}, \mathrm{p}}^{g}$ & $\Gamma_{\mathrm{R}, \mathrm{c}}^{e}$ & $\Gamma_{\mathrm{R}, \mathrm{c}}^{g}$ & $\Gamma_{\mathrm{L}, \mathrm{p}}^{e}$ & $\Gamma_{\mathrm{L}, \mathrm{p}}^{g}$ & $\Gamma_{\mathrm{L}, \mathrm{c}}^{e}$ & $\Gamma_{\mathrm{L}, \mathrm{c}}^{g}$ & & & & \\
\hline $1 \mathrm{e}$ & $4.52 \times 10^{7}$ & $4.79 \times 10^{-6}$ & $4.65 \times 10^{-6}$ & 1110 & 1070 & 1380 & 1500 & $9.05 \times 10^{6}$ & $1.09 \times 10^{6}$ & 0.0357 & $1 \mathrm{GHz}$ & $72 \mathrm{kHz}$ & $31.2 \mathrm{MHz}$ \\
\hline $2 \mathrm{e}$ & 2970 & $2.35 \times 10^{-10}$ & $2.31 \times 10^{-10}$ & 0.437 & 0.43 & $6.97 \times 10^{-3}$ & $5.46 \times 10^{-3}$ & $1.01 \times 10^{-2}$ & $8.07 \times 10^{-3}$ & 0.907 & $50 \mathrm{GHz}$ & $53 \mathrm{kHz}$ & $15.6 \mathrm{MHz}$ \\
\hline
\end{tabular}

TABLE I. Qubit types, working points, and information-loss time scales due to different mechanisms in the P:Si dot-donor system. $\Gamma_{\mathrm{R}}$ : relaxation rate, $\Gamma_{\mathrm{L}}$ : leakage rate, $\Gamma_{2}^{*}$ dephasing rate. Lower index "p" ("c") refers to phonons $(1 / f$ charge noise). For relaxation and leakage processes, the initial state can be the qubit ground state $|g\rangle$ or the qubit excited state $|e\rangle$. Rates are evaluated for temperature $T=50 \mathrm{mK}$.

experiments ${ }^{25,26}$ on state-of-the-art silicon quantum devices have shown pronounced significance of $1 / f$ charge noise. By comparing various information-loss mechanisms, we will conclude that the most relevant one is dephasing due to $1 / f$ charge noise.

To describe dephasing, in this section, we treat the $1 / f$ charge noise as a time-dependent on-site energy difference $\delta U(t)$ between the interface and the donor felt by the electron. Correspondingly, the noise Hamiltonian reads

$$
H_{\text {noise }}=\frac{\delta U(t)}{2} \sigma_{z} .
$$

We describe the dynamics of the nuclear-spin qubit under the influence of this noise using the effective qubit Hamiltonian, obtained by projecting the total Hamiltonian $H_{\text {tot }}=H+H_{\text {noise }}$ on the qubit subspace:

$$
H_{\mathrm{q}}=P H_{\mathrm{tot}} P=\frac{h f_{\mathrm{L}}}{2} \sigma_{z}^{\prime}+\frac{\delta U(t)}{2} \mathcal{L} \sigma_{z}^{\prime}+\frac{\delta U(t)}{2} \mathcal{T} \sigma_{x}^{\prime}(5)
$$

where $P=|e\rangle\langle e|+| g\rangle\langle g|$, and the qubit Larmor frequency $f_{\mathrm{L}}$, the longitudinal matrix element $\mathcal{L}$, and the transverse matrix element $\mathcal{T}$, are defined via

$$
\begin{aligned}
h f_{\mathrm{L}} & =\epsilon_{e}-\epsilon_{g}, \\
\mathcal{L} & =\frac{\left\langle e\left|\sigma_{z}\right| e\right\rangle-\left\langle g\left|\sigma_{z}\right| g\right\rangle}{2}, \\
\mathcal{T} & =\left\langle e\left|\sigma_{z}\right| g\right\rangle .
\end{aligned}
$$

Furthermore, the Pauli matrices are defined in the twodimensional qubit subspace, e.g., $\sigma_{z}^{\prime}=|e\rangle\langle e|-| g\rangle\langle g|$. In general, both the longitudinal $\mathcal{L}$ and transverse $\mathcal{T}$ matrix elements are finite. However, for describing dephasing, it is a good approximation to disregard $\mathcal{T}$ in our setup, and keep only $\mathcal{L}$; this is what we do from now on. The validity of this approximation is discussed in section $\mathrm{V}$.

We assume that the noise is classical, Gaussian ${ }^{27,28}$ and has the $1 / f$ power spectrum ${ }^{25,29-31}$

$$
S_{\delta U}(f) \equiv \int_{-\infty}^{\infty} d t e^{i 2 \pi f t} \overline{\delta U(t) \delta U(0)}=\frac{\alpha_{1 / f} k_{B} T}{2 \pi f},
$$

where the overline denotes the average over the noise realizations, $\alpha_{1 / f}$ denotes the overall strength of the noise, $k_{B}$ is the Boltzmann constant, and $T$ is the temperature. From the analysis of Refs. 14 (Ref. 26), we estimate $\alpha_{1 / f} \approx 43.5 \mathrm{neV}\left(\alpha_{1 / f} \approx 5.1 \mathrm{neV}\right)$, see Appendix A. In what follows, we will take the greater value for $\alpha_{1 / f}$ to obtain quantitative results.

In the description of dephasing due to $1 / f$ noise, the experimental integration time $t_{i}$ is often taken into account ${ }^{27}$. For example, the decay of the qubit polarization in a Ramsey-type experiment ${ }^{6}$ is inferred by doing many measurement cycles: there are many grid points along the waiting-time axis, and for each waiting time, many measurement cycles are carried out to obtain reliable statistics. The integration time $t_{i}$ is the total time required to carry out all these measurement cycles; in our 
estimates, we will assume that the order of magnitude of this integration time is a second. The finite integration time implies that the slow noise components, i.e., those that can be regarded constant during the integration time, will not be able to influence the experiment. This argument implies that dephasing is insensitive to the low-frequency part of the noise spectrum, that is, the part of the spectrum below the integration frequency $f_{i}=1 / t_{i}$ can be neglected.

With the above assumptions, dephasing can be characterized by an approximately Gaussian decay ${ }^{27}$, i.e., the length of the qubit polarization vector decays in time as $\sim e^{-\left(\Gamma_{2}^{*} t\right)^{2}}$, with the inhomogeneous dephasing rate given by

$$
\Gamma_{2}^{*} \approx \frac{E_{1 / f}}{h} \sqrt{2 \pi \ln \frac{E_{1 / f}}{h f_{i}}}
$$

where we have introduced $E_{1 / f}=\sqrt{\alpha_{1 / f} k_{B} T} \mathcal{L}$. The energy scale $E_{1 / f}$ can be expressed from the above numerical estimate of the noise strength $\alpha_{1 / f}$; assuming $T=50$ $\mathrm{mK}$, we find $E_{1 / f} \approx h \times \mathcal{L} \times 0.1 \mathrm{GHz}$. Note that a necessary formal condition for the approximations leading to Eq. (8) is $\frac{E_{1 / f}}{h f_{i}} \gtrsim 10$. In practice, this condition always holds (as long as this is the dominant dephasing mechanism), since it is translated by Eq. (8) to $\Gamma_{2}^{*} / f_{i} \gtrsim 40$, and the latter holds because in any reasonable dephasing-time measurement, the integration time is orders of magnitude larger than the dephasing time itself.

We evaluate the result Eq. (8) for a particular parameter set (see Table I) using the nuclear-spin basis states obtained by the numerical diagonalization of $H$ of Eq. (2) and the subsequent evaluation of $\mathcal{L}$ from Eq. (6b). Our numerical estimate for this particular working point is $\Gamma_{2}^{*}=45.2 \mathrm{MHz}$, a rate that is much larger than the esimated electrically induced Rabi frequency $f_{\text {Rabi }}=72$ $\mathrm{kHz}$ at this working point. (For the Rabi frequency estimate, see Ref. 13.) This is a strong indication that the nuclear spin cannot be used as a qubit in this working point. One way to make this a useful qubit is to reduce the strength of $1 / f$ noise by at least 4 orders of magnitude.

The reason for this strong dephasing is as follows. Recall that the working point studied here is the ionization point. Here, a weak electrical perturbation can displace the electron along the dot-donor (y) direction very effectively, which implies a significant change in the electron density on the donor, which in turn implies a significant change in the Knight shift felt by the nuclear spin. This mechanism was implicitly quantified already in Eq. (5) of Ref. 13, see the third component of $\boldsymbol{b}_{\mathrm{ac}}$ therein.

\section{Nuclear-spin qubit and its dephasing with two donor electrons}

In contrast to the 1e setup showing poor coherence properties, much improvement is anticipated for the 2e $\operatorname{setup}^{13}$. Importantly, silicon-based dot-donor devices holding an even number of electrons are available experimentally $^{21,32}$. Here we introduce the model Hamiltonian for this system following Ref. 13, recall how electrical control of the nuclear-spin qubit is envisioned, and determine the dephasing rate of the nuclear-spin qubit due to $1 / f$ charge noise.

The two electrons in the dot-donor system can fill the orbitals $|i\rangle$ and $|d\rangle$, both providing two sublevels due to the electron spin. This implies that there are six twoelectron states to take into account in a minimal model. We use the standard basis set $\left|S_{20}\right\rangle,|S\rangle,\left|T_{+}\right\rangle,\left|T_{0}\right\rangle,\left|T_{-}\right\rangle$, $\left|S_{02}\right\rangle$. Here, the first [last] element is the spin singlet state in which both electrons are localized at the interface [donor], also referred to as the $(2,0)[(0,2)]$ charge configuration. Furthermore, the remaining four states are the standard singlet and triplet states in which one electron is localized at each site, also referred to as the $(1,1)$ charge configuration.

We assume that the on-site Coulomb repulsion $U_{C}$ between the electrons $(\sim \mathrm{meV})$ is much larger then the tunnel coupling $V_{t}(\sim 10-100 \mu \mathrm{eV})$. In the $2 \mathrm{e}$ setup, we consider the case when the on-site energy difference $U$ is set such that on-site energies of the four $(1,1)$ states and the single $(0,2)$ state are close to each other, and we restrict our attention to the dynamics in this low-energy electronic subspace. That is, we neglect the high-energy $\left|S_{20}\right\rangle$ state, and model the system by a ten-dimensional Hamiltonian, using the five electronic and the two nuclear-spin basis states.

The Hamiltonian describing this arrangement is the two-electron version of the single-electron Hamiltonian introduced in Eq. (2). The terms which are different from the single-electron case read:

$$
\begin{aligned}
H_{\mathrm{ch}} & =-\tilde{U}\left|S_{02}\right\rangle\left\langle S_{02}\right|+\frac{V_{t}}{\sqrt{2}}\left(|S\rangle\left\langle S_{02}\right|+h . c .\right), \\
H_{B, \mathrm{e}} & =h \gamma_{\mathrm{e}} B\left(\left|T_{+}\right\rangle\left\langle T_{+}|-| T_{-}\right\rangle\left\langle T_{-}\right|\right), \\
H_{\mu, \mathrm{e}} & =h \gamma_{\mathrm{e}} \frac{\beta d}{2 \sqrt{2}}\left(\left|T_{-}\right\rangle\left\langle S|-| T_{+}\right\rangle\langle S|\right)+\text { h.c. },
\end{aligned}
$$

where $\tilde{U}=U-U_{C}$ is the energy detuning measured from the $(1,1)-(0,2)$ tipping point $U=U_{C}$. Each terms above acts as the identity on the nuclear spin. Furthermore, the hyperfine Hamiltonian takes the form

$$
\begin{aligned}
H_{\mathrm{hf}}= & \frac{A}{2}\left(\left|T_{+}\right\rangle\left\langle T_{+}|-| T_{-}\right\rangle\left\langle T_{-}\right|\right. \\
& \left.-|S\rangle\left\langle T_{0}|-| T_{0}\right\rangle\langle S|\right) I_{z} \\
& +\frac{A}{2 \sqrt{2}}\left[\left(|S\rangle\left\langle T_{+}|+| T_{0}\right\rangle\left\langle T_{+}\right|\right.\right. \\
& \left.\left.+\left|T_{-}\right\rangle\left\langle T_{0}|-| T_{-}\right\rangle\langle S|\right) I_{+}+\text {h.c }\right] .
\end{aligned}
$$

Note that in Eq. (10), we have corrected a few typos that appeared in Eq. (A2b) of Ref. 13.

Following the 1e case, it is useful to introduce the antibonding and bonding singlet energy eigenstates of $H_{\mathrm{ch}}$, i.e., the molecular states formed by $|S\rangle$ and $\left|S_{02}\right\rangle$, which we will denote as $\left|S_{a}\right\rangle$ and $\left|S_{b}\right\rangle$, respectively. 
We plot the ten energy eigenvalues of this model in Fig 1c, as a function of the detuning parameter $\tilde{U}$, for homogeneous magnetic field $B=906.5 \mathrm{mT}$, tunnelling amplitude $V_{t} / h=50 \mathrm{GHz}$, magnetic-field gradient $\beta=$ $0.47 \mathrm{mT} / \mathrm{nm}$, and donor-interface distance $d=15 \mathrm{~nm}$. Here again, we define the nuclear-spin qubit basis states as the lowest-energy eigenstates: $|e\rangle_{2 e} \equiv\left|S_{b} \Downarrow\right\rangle$ and $|g\rangle_{2 e} \equiv\left|S_{b} \Uparrow\right\rangle$.

Before presenting our results for the dephasing time caused by $1 / f$ charge noise, we recall that an important energy scale for the nuclear-spin qubit is the energy gap between the electronic states $\left|S_{b}\right\rangle$ and $\left|T_{-}\right\rangle$, see Fig. 1c. We denote the value of this gap at zero detuning $\tilde{U}=0$, as obtained from the electronic Hamiltonian $H_{\mathrm{ch}}+H_{B, \mathrm{e}}$, by $\delta$. It can be expressed as

$$
\delta=\frac{V_{t}}{\sqrt{2}}-h \gamma_{\mathrm{e}} B .
$$

To ensure that the nuclear-spin qubit dynamics upon electrical drive follows regular and fast Rabi oscillations ${ }^{13}$, it is reasonable to set the value of $\delta$ much larger than the coupling matrix element induced by the inhomogeneous magnetic field between the electronic states $\left|S_{b}\right\rangle$ and $\left|T_{-}\right\rangle$. This relation is satisfied, e.g., with the choice $\delta=200\left\langle S_{b}\left|H_{\mu, \mathrm{e}}\right| T_{-}\right\rangle$. The parameter values given above satisfy this relation, since they correspond to $\left\langle S_{b}\left|H_{\mu, \mathrm{e}}\right| T_{-}\right\rangle \approx 50 \mathrm{MHz}$ and $\delta \approx 10 \mathrm{GHz}$.

In the $2 \mathrm{e}$ setup, the noise Hamiltonian, derived from its 1e counterpart in Eq. (4), reads

$$
H_{\text {noise }}=-\delta U(t)\left|S_{02}\right\rangle\left\langle S_{02}\right| \text {. }
$$

Projecting the total Hamiltonian onto the twodimensional subspace of the nuclear-spin qubit, as done for the 1e case in Eq. (5), we obtain the right hand side of Eq. (5) with the following identifications:

$$
\begin{aligned}
h f_{\mathrm{L}} & =\epsilon_{e}-\epsilon_{g}, \\
\mathcal{L} & =\left|\left\langle g \mid S_{02}\right\rangle\right|^{2}-\left|\left\langle e \mid S_{02}\right\rangle\right|^{2}, \\
\mathcal{T} & =-2\left\langle e \mid S_{02}\right\rangle\left\langle S_{02} \mid g\right\rangle .
\end{aligned}
$$

We obtain the dephasing rate $\Gamma_{2}^{*}$ from Eq. (8), after evaluating the longitudinal matrix element $\mathcal{L}$ in Eq. (13b) with the numerically obtained energy eigenstates $|g\rangle$ and $|e\rangle$. For the above parameter values, we find $\Gamma_{2}^{*} \approx$ $2.97 \mathrm{kHz}$, see Table I. Note that this rate is significantly smaller than the Rabi frequency in Table I (estimated in Ref. 13), suggesting that the nuclear spin can be used as a functional qubit in this setting.

Now, we argue that this dephasing is a consequence of the hyperfine interaction, and not influenced significantly by the inhomogeneous magnetic field. This is revealed by a perturbative approach that yields the analytical result for the longitudinal coupling strength

$$
\begin{aligned}
\mathcal{L}= & \frac{A^{2}}{32 \delta^{2}}+\frac{2 A^{2}}{32\left(2 \Delta_{Z}+2 \delta\right) \delta} \\
& -\frac{2 A^{2}}{32\left(2 \Delta_{Z}+\delta\right)\left(2 \Delta_{Z}+2 \delta\right)}-\frac{A^{2}}{32\left(2 \Delta_{Z}+\delta\right)^{2}},
\end{aligned}
$$

with $\Delta_{Z}=h \gamma_{\mathrm{e}} B$ and correspondingly, $\Gamma_{2}^{*} \approx 2.77 \mathrm{kHz}$ in the working point defined above. The result (14) depends on the hyperfine coupling strength $A$, but does not depend on the magnetic-field gradient $\beta$.

To obtain Eq. (14), we take $H_{0}=H_{\mathrm{ch}}+H_{B, \mathrm{e}}+H_{B, \mathrm{n}}$ as the unperturbed Hamiltonian, take $H_{1}=H-H_{0}-H_{\mu, \mathrm{n}}$ as the perturbation, and neglect the small term $H_{\mu, \mathrm{n}}$ for simplicity. Then, we apply time-independent thirdorder perturbation theory to calculate the perturbationinduced change in the energy splitting of the nuclear-spin qubit states $\left|S_{b} \Downarrow\right\rangle$ and $\left|S_{b} \Uparrow\right\rangle$. According to Eq. (5), we identify this change with $\delta U \mathcal{L}$, express $\mathcal{L}$, and use $h \gamma_{\mathrm{n}} B \ll h \gamma_{\mathrm{e}} B, V_{t}, \delta$ to obtain Eq. (14).

The perturbative result (14) for dephasing mechanism is visualized in the level diagram shown in Fig. 3. The blue horizontal lines depict the energy levels of the unperturbed Hamiltonian. Arrows represent relevant perturbation matrix elements that contribute to dephasing. In the third-order formula (14), each of the four terms can be associated to a three-step loop drawn by the perturbation matrix elements. For example, the loop corresponding to the first term is $\left|S_{b} \Downarrow\right\rangle_{0} \rightarrow\left|S_{b} \Downarrow\right\rangle_{0} \rightarrow\left|T_{-} \Uparrow\right\rangle_{0} \rightarrow$ $\left|S_{b} \Downarrow\right\rangle$.

To extend the perturbative formula (14) of $\mathcal{L}$ for the case of non-zero $\tilde{U}$, we use the exact eigenvalues and eigenstates of (9a), yielding

$$
\begin{aligned}
\mathcal{L}= & \frac{2 V_{t}^{2}}{\tilde{U}^{2}+2 V_{t}^{2}}\left[\frac{A^{2}}{32 \delta^{\prime 2}}+\frac{2 A^{2}}{32\left(2 \Delta_{Z}+2 \delta^{\prime}\right) \delta^{\prime}}\right. \\
& \left.-\frac{2 A^{2}}{32\left(2 \Delta_{Z}+\delta^{\prime}\right)\left(2 \Delta_{Z}+2 \delta^{\prime}\right)}-\frac{A^{2}}{32\left(2 \Delta_{Z}+\delta^{\prime}\right)^{2}}\right],
\end{aligned}
$$

where $\delta^{\prime}=\frac{\tilde{U}}{2}+\frac{\sqrt{\tilde{U}^{2}+2 V_{t}^{2}}}{2}-\Delta_{Z}$. In comparison to Eq. (14), the energy denominators are different in (15). The prefactor in Eq. (15) depends on the squared detuning parameter $\tilde{U}^{2}$, i.e., this prefactor is suppressed if we move away from the working point from $\tilde{U}=0$ either to the positive or negative direction. For the negative [positive] direction, this dephasing suppression is dominated by the feature that the electronic state acquires a growing weight in the $(1,1)[(0,2)]$ charge configuration, and hence gets less affected by noise (hyperfine interaction).

In Fig. 2a, we plot the numerically calculated dephasing rate $\Gamma_{2}^{*}$ as a function of the energy detuning $\tilde{U}$ and the magnetic field $B$. In the figure, the above working point is denoted by an ' $x$ '. Note that in the figure, the tunnel matrix element $V_{t}$ is changed together with the magnetic field $B$ such that $\delta$ is kept fixed, see Eq. (11). The most relevant features in Fig. 2a are as follows.

(i) For $B \gtrsim 0.4 \mathrm{~T}$, the dephasing rate decreases as the energy detuning $\tilde{U}$ increases, and the dephasing rate is hardly dependent on the magnetic field. (ii) These trends are confirmed by the perturbative result (15). For example, a comparison of the numerical and perturbative result, along a horizontal cut of Fig. 2a containing the working point ' $x$ ', is shown in Fig. 2b. Using Eq. (15), we can explain that the decreasing trend of the dephasing 

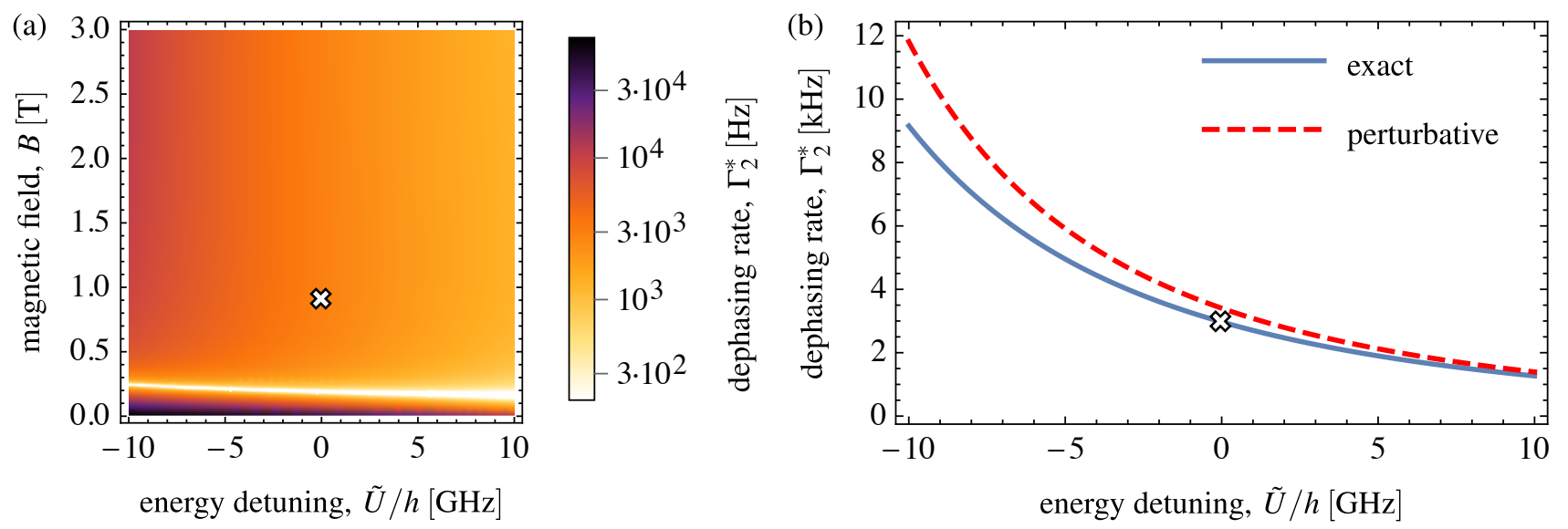

FIG. 2. Dephasing rate induced by $1 / f$ charge noise in the two-electron setup. (a) Numerically calculated dephasing rate as a function of the $(1,1)-(0,2)$ energy detuning and the magnetic field. Note that the tunnel matrix element $V_{t}$ is tied to the magnetic field $B$ to ensure a constant $S-T_{-}$energy gap at $\tilde{U}=0$, via $V_{t}=\sqrt{2}\left(\delta+h \gamma_{\mathrm{e}} B\right)$ (cf. Eq. (11)). The white cross denotes the working point $B=906.5 \mathrm{mT}, \tilde{U}=0$ and $\delta / h=10 \mathrm{GHz}$. (b) Exact numerical result (solid) is compared to the perturbative result of Eq. (15) at the working-point magnetic field $B=906.5 \mathrm{mT}$. Further parameters: $\alpha_{1 / f}=43.5 \mathrm{neV}$, $T=50 \mathrm{mK}$.

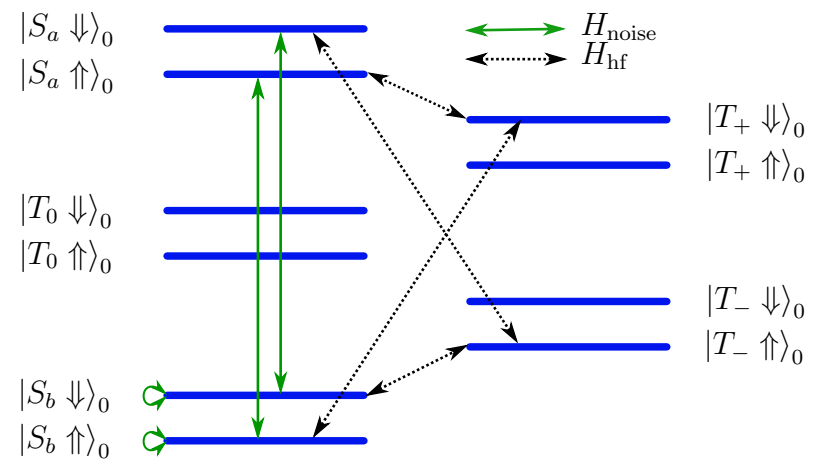

FIG. 3. Level diagram of the hyperfine states in the twoelectron setup. Horizontal lines indicate the eigenstates of the unperturbed Hamiltonian $H_{\mathrm{ch}}+H_{B, \mathrm{e}}+H_{B, \mathrm{n}}$. Arrows indicate the perturbation matrix elements relevant for the dephasing in the specific working point (see text). Perturbation matrix elements that are not relevant for this dephasing mechanism are not shown.

rate with increasing energy detuning $\tilde{U}$ is mostly due to the increasing energy gap between the electronic singlet ground state and the electronic excited states, cf. Fig. 1c. (iii) For magnetic fields much weaker than the working point value, the dephasing rate does depend significantly on $B$. This regime is beyond the validity of the perturbative result (15) due the smallness of the magnetic field.

In conclusion, we have evaluated the inhomogeneous dephasing rate of the nuclear-spin qubit for a P:Si 1e and 2e dot-donor setup subject to (artificial) spin-orbit coupling, and identified a parameter range for the 2e setup where the dephasing time is much longer than the time required for single-qubit operations.

\section{RELAXATION AND LEAKAGE DUE TO 1/F CHARGE NOISE}

Besides dephasing discussed above, the presence of $1 / f$ charge noise also opens up channels for information loss. Here, we focus on the 2e setup, and describe two types of inelastic processes caused by $1 / f$ charge noise, see Fig. 4 . First, we consider inelastic processes between the two qubit basis states, denoted as $\Gamma_{\mathrm{R}}^{e} \equiv \Gamma^{g e}$ and $\Gamma_{\mathrm{R}}^{g} \equiv \Gamma^{e g}$ in Fig. 4 , to be referred to as relaxation. Second, we consider inelastic processes that bring the system from one of the qubit basis states to a state outside the qubit's Hilbert space, to be referred to as leakage, shown as $\Gamma^{f g}$ and $\Gamma^{f e}$ in Fig. 4. Our conclusion is that the time scales of these processes in the vicinity of the working point are much longer than the dephasing time $1 / \Gamma_{2}^{*}$ derived in the previous section, hence they hardly affect the functionality of the nuclear-spin qubit.

We use the qubit Hamiltonian $H_{\mathrm{q}}$ of Eq. (5) to describe the relaxation processes, with the adjustment that $\delta U$ is treated now as an operator representing the environment producing the $1 / f$ noise. Recall that the longitudinal and transverse coupling matrix elements $\mathcal{L}$ and $\mathcal{T}$ are evaluated for the 1e [2e] setup via Eq. (6) [Eq. (13)]. According to Bloch-Redfield theory ${ }^{27}$, the downhill and uphill relaxation rates are given by

$$
\begin{aligned}
& \Gamma_{\mathrm{R}}^{e}=\frac{1}{2 \hbar^{2}} \mathcal{T}^{2} \frac{1+n_{\mathrm{BE}}\left(h f_{\mathrm{L}}\right)}{1+2 n_{\mathrm{BE}}\left(h f_{\mathrm{L}}\right)} S_{\delta U}\left(f_{\mathrm{L}}\right), \\
& \Gamma_{\mathrm{R}}^{g}=\frac{1}{2 \hbar^{2}} \mathcal{T}^{2} \frac{n_{\mathrm{BE}}\left(h f_{\mathrm{L}}\right)}{1+2 n_{\mathrm{BE}}\left(h f_{\mathrm{L}}\right)} S_{\delta U}\left(f_{\mathrm{L}}\right) .
\end{aligned}
$$

Here, $n_{\mathrm{BE}}\left(h f_{\mathrm{L}}\right)$ is the temperature-dependent BoseEinstein function, and $S_{\delta U}(f)$ is the symmetrized noise power spectrum of the operator $\delta U$, which is given by 


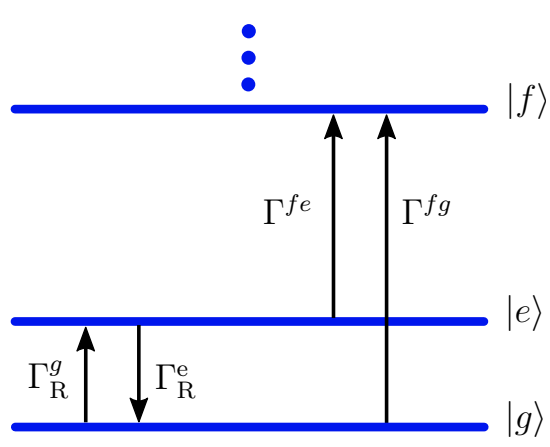

FIG. 4. Inelastic transitions due to $1 / f$ charge noise and phonons. Downhill and uphill processes within the qubit subspace contribute to qubit relaxation. Transitions from one of the qubit basis states $|g\rangle$ or $|e\rangle$ to a state outside of the qubit subspace cause leakage.

the same formula (7) as in the classical case.

For the $2 \mathrm{e}$ setup, the numerical values of the $1 / f$ induced relaxation rates of the nuclear-spin qubit are around $1 \mathrm{~Hz}$, see Table I. This implies that relaxation due to $1 / f$ noise is much less relevant than dephasing. Note that the uphill and downhill relaxation rates are almost the same, in line with the fact that the thermal frequency scale $k_{B} T / h \approx 1.04 \mathrm{GHz}$ well exceeds the qubit splitting $f_{\mathrm{L}} \approx 14.5 \mathrm{MHz}$ in this point.

The transverse matrix element can be obtained from perturbation theory similarly as in the case of dephasing.

One possible way for the derivation is to apply quasiquasidegenerate perturbation theory ${ }^{33}$ to obtain an effective Hamiltonian for the nuclear-spin qubit subspace, identify the off-diagonal element of that Hamiltonian with $\delta U \mathcal{T} / 2$ according to Eq. (5), and express $\mathcal{T}$ from that equation. From this approach, for the tipping point $\tilde{U}=0$, we obtain

$$
\begin{aligned}
\mathcal{T} & =\frac{A\left(h \gamma_{e} \beta d\right)}{16}\left(\frac{1}{\delta^{2}}+\frac{1}{\left(2 \Delta_{Z}+\delta\right)^{2}}\right. \\
& \left.+\frac{2}{\left(2 \Delta_{Z}+2 \delta\right) \delta}+\frac{2}{\left(2 \Delta_{Z}+2 \delta\right)\left(2 \Delta_{Z}+\delta\right)}\right) .
\end{aligned}
$$

The value obtained from this formula is $\mathcal{T} \approx 1.96 \times 10^{-5}$, in good agreement with the numerical result $\mathcal{T}=1.98 \times$ $10^{-5}$

The perturbative contributions can again be visualized by drawing the perturbation matrix elements as steps between the energy levels of the unperturbed Hamiltonian; this is shown in Fig. 5. The four terms in the perturbative formula (17) correspond to six three-step paths in Fig. 5 connecting the two qubit basis states. For example, the first term of (17) corresponds to the path $\left|S_{b} \Downarrow\right\rangle_{0} \rightarrow\left|S_{b} \Downarrow\right\rangle_{0} \rightarrow\left|T_{-} \Uparrow\right\rangle_{0} \rightarrow\left|S_{b} \Uparrow\right\rangle_{0}$. As seen in Fig. 5, all three-step paths connecting the two qubit basis states contain one hyperfine matrix element and one inhomogeneous magnetic field matrix element (besides one noise matrix element), hence we conclude that relaxation

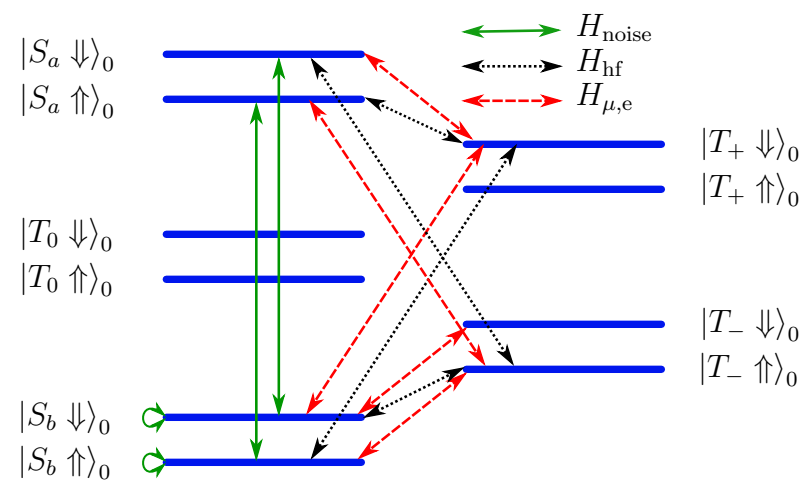

FIG. 5. Level diagram of the hyperfine states in the twoelectron setup. Horizontal lines indicate the eigenstates of the unperturbed Hamiltonian $H_{\mathrm{ch}}+H_{B, \mathrm{e}}+H_{B, \mathrm{n}}$. Arrows indicate the perturbation matrix elements involved in relaxation and leakage (see text).

in this case is dominated by the interplay of hyperfine interaction and the inhomogeneous magnetic field.

Leakage rates, i.e., noise-induced transition rates from the nuclear-spin qubit basis states toward higher-lying eigenstates (see Fig. 4), can also be described by the Bloch-Redfield result. For example, the leakage from the qubit ground state $|g\rangle$ has the rate

$$
\begin{aligned}
\Gamma_{\mathrm{L}}^{g} & =\sum_{f \neq g, e} \Gamma^{f g} \\
& =\frac{1}{2 \hbar^{2}} \sum_{f \neq g, e} \mathcal{T}_{f g}^{2} \frac{n_{\mathrm{BE}}\left(\epsilon_{f g}\right)}{1+2 n_{\mathrm{BE}}\left(\epsilon_{f g}\right)} S_{\delta U}\left(\epsilon_{f g} / h\right) .
\end{aligned}
$$

Here, the sum goes for the possible higher-lying final states $|f\rangle$, the matrix element is $\mathcal{T}_{f g}=\left\langle f\left|\sigma_{z}\right| g\right\rangle$ for the $1 \mathrm{e}$ case and $\mathcal{T}_{f g}=-2\left\langle f \mid S_{02}\right\rangle\left\langle S_{02} \mid g\right\rangle$ for the $2 \mathrm{e}$ case, and $\epsilon_{f g}$ is the distance between the energies of $|f\rangle$ and $|g\rangle$. The leakage rate $\Gamma_{L}^{e}$ for the qubit excited state $|e\rangle$ is expressed analogously to Eq. (18).

As seen in Table I, the leakage rates for the 2e setup in the working point are of the order of $0.01 \mathrm{~Hz}$, slower and hence less significant than the previously considered processes. At this working point, the dominant leakage process from $|g\rangle$ is the one toward $\left|T_{-} \Uparrow\right\rangle$. Approximating the leakage-rate sum in Eq. (18) with this single contribution, expressing $\mathcal{T}_{f g}$ using perturbation theory, and using the relation $\delta \ll h \gamma_{\mathrm{e}} B$, we find

$$
\Gamma_{\mathrm{L}}^{g} \approx \frac{1}{2 \hbar^{2}} \frac{\left(h \gamma_{e} \beta d\right)^{2}}{8 \delta^{2}} \frac{1}{e^{\left(\delta / k_{B} T\right)}+1} \frac{\alpha_{1 / f} k_{B} T}{\delta} .
$$

According to this formula, leakage is dominated by the magnetic-field gradient $\beta$. Similar considerations lead to $\Gamma_{L}^{e} \approx \Gamma_{L}^{g}\left[1+\left(\frac{A}{h \gamma_{e} \beta d}\right)^{2}\right]$, i.e., that the leakage from the excited state has an additional contribution from the hyperfine interaction $A$. These results are in line with the level diagram shown in Fig. 5, where the qubit ground state is hybridized with a single $T_{-}$sublevel, whereas the qubit excited state is hybridized with both $T_{-}$sublevels. 


\section{RELAXATION AND LEAKAGE DUE TO PHONONS}

Besides charge noise, phonon absorption or emission can also cause transitions between the energy levels. Here we consider the deformation-potential electron-phonon interaction mechanism ${ }^{34,35}$. This mechanism is enhanced in a silicon dot-donor electron system (compared to, e.g., a double-dot or double-donor setup) due to the different valley compositions of the electronic states in the dot and the donor ${ }^{23}$. For the energy distances considered here, only long-wavelength acoustic phonons have to be considered.

We describe the phonon-mediated inelastic transitions using Bloch-Redfield theory, similarly to the case of charge-noise-mediated transitions in section III. As a natural consequence, the phonon-induced transition rates are related to the charge-noise-induced transitions rates by the relative weight of the noise spectral densities at the transition frequency. For example, the phonon-mediated downhill relaxation rate is

$$
\Gamma_{\mathrm{R}, \mathrm{p}}^{e}=\frac{S_{\mathrm{ph}}\left(f_{L}\right)}{S_{\delta U}\left(f_{L}\right)} \Gamma_{\mathrm{R}, \mathrm{c}}^{e},
$$

and analogous relations hold for the phonon-mediated uphill relaxation rate $\Gamma_{\mathrm{R}, \mathrm{p}}^{g}$ and the leakage rates $\Gamma_{\mathrm{L}, \mathrm{p}}^{e}$ and $\Gamma_{\mathrm{L}, \mathrm{p}}^{g}$, with the caveat that $f_{L}$ in Eq. (20) has to be replaced with the corresponding transition frequencies.

To obtain the noise spectral density $S_{\mathrm{ph}}\left(f_{L}\right)$, representing the phonons, one starts from the single-electron electron-phonon interaction Hamiltonian ${ }^{23}$ for the dotdonor system:

$$
H_{\mathrm{eph}}=\frac{\hat{U}_{\mathrm{ph}}}{2} \sigma_{z}
$$

where $\hat{U}_{\mathrm{ph}}$ is expressed via phonon creation and annihilation operators in Eqs. (12) and (13) of Ref. 23. The symmetrized phonon noise density is then expressed as (cf. Eq. (7))

$$
S_{\mathrm{ph}}(f)=\frac{1}{2} \int_{-\infty}^{\infty} d t e^{i 2 \pi f t} \overline{\left\{\hat{U}_{\mathrm{ph}}(t), \hat{U}_{\mathrm{ph}}(0)\right\}},
$$

where the time-dependent operators are defined in the interaction picture, the curly brackets $\{.,$.$\} denote the$ anticommutator, and the overline denotes thermal average for the equilibrium phonon bath ${ }^{36}$.

Equation (22) is evaluated as

$$
\begin{aligned}
& S_{\mathrm{ph}}(f)=\left[1+2 n_{\mathrm{BE}}(h f)\right] S_{\mathrm{ph}}^{(0)}(f), \\
& S_{\mathrm{ph}}^{(0)}(f)=\frac{\Xi^{2} \hbar}{30 \pi \rho}\left(\frac{2}{3 v_{\mathrm{L}}^{5}}+\frac{1}{v_{\mathrm{T}}^{5}}\right)(2 \pi f)^{3} .
\end{aligned}
$$

Here, the material-specific parameters for silicon are the uniaxial deformation potential parameter $\Xi_{u}=8.77 \mathrm{eV}$, the mass density $\rho=2330 \mathrm{~kg} / \mathrm{m}^{3}$, and the longitudinal and transverse sound velocities $v_{\mathrm{L}}=9330 \mathrm{~m} / \mathrm{s}$ and $v_{\mathrm{T}}=$ $5420 \mathrm{~m} / \mathrm{s}$.
The results obtained for the $1 / f$ charge noise model can therefore be converted to the case of phononmediated relaxation and leakage using Eqs. (23) and (20). As seen in Table I, the phonon-induced relaxation rates are approximately 10 orders of magnitude smaller then the relaxation rates due to $1 / f$ charge noise. In contrast, the leakage rates corresponding to processes induced by phonons and $1 / f$ charge noise are very similar. We emphasize that all of these leakage and relaxation rates are smaller than the dephasing rate.

\section{DISCUSSION}

Artifical vs intrinsic spin-orbit interaction. Ref. 23 suggests that electrical control of the nuclear-spin qubit should be possible either by relying on an inhomogeneous magnetic field (artificial spin-orbit interaction), or by relying on intrinsic spin-orbit interaction. In a simple phenomenological picture, spin-orbit interaction can influence the dot-donor system in two ways; both effects have been observed in silicon double quantum dots ${ }^{37-40}$. On the one hand, it renormalizes the g-factor (with few percents), potentially making it anisotropic and different at the donor and in the dot. On the other hand, it induces a spin-dependent interdot tunnelling matrix element (few tens of $\mathrm{MHz}$ ). The consequences of the anisotropic and different g-factors are similar to those of the inhomogeneous magnetic field. The consequences of the spin-dependent tunneling term are expected to be qualitatively different. For example, while the inhomogeneous magnetic field provides matrix elements within the $(1,1)$ charge configuration, e.g., between $|S\rangle$ and $\left|T_{-}\right\rangle$(see Eq. (9c)), spin-dependent tunnelling provides a matrix element connecting $(1,1)$ states with $\left|S_{02}\right\rangle$. Nevertheless, in the vicinity of the $(1,1)$ - $(0,2)$ tipping point, where the singlet electronic ground state $\left|S_{g}\right\rangle$ is a balanced superposition of $(1,1)$ and $(0,2)$ charge states, we expect that the dynamics induced by spin-orbit interaction is similar to that induced by an inhomogeneous magnetic field.

Neglecting the transverse noise term in the dephasing model. We calculated the nuclear-spin qubit dephasing time due to $1 / f$ charge noise in section II. For the dephasing calculation, we disregarded the transverse noise term with the prefactor $\mathcal{T}$. This simplification is justified as long as the noisy component of the Larmor frequency is dominated by the longitudinal component proportional to $\mathcal{L}$; formally, that condition reads $\mathcal{T}^{2} \delta U /\left(2 h f_{L} \mathcal{L}\right) \ll 1$. For a rough estimate of the importance of $\mathcal{T}$ in dephasing, we take $\delta U=1 \mu \mathrm{eV}$, yielding $\mathcal{T}^{2} \delta U /\left(2 h f_{L} \mathcal{L}\right) \approx 6.70 \times 10^{-4}$ for the working point of the $2 \mathrm{e}$ setup shown in Fig. 2a, and thereby suggesting that our result is accurate in the vicinity of the working point. Note, however that for low magnetic field, far from the working point, the longitudinal matrix element $\mathcal{L}$ vanishes, see the horizontal white stripe for $B \approx 0.2 \mathrm{~T}$ in Fig. 2a. In this region, the description of dephasing should be refined ${ }^{41}$. 
Leakage due to uphill charge transitions. For the 2e setup, we have described information loss at a welldefined working point specified in, e.g., Table I. Departing from this example working point might provide optimized results for certain target quantities, e.g., the qubit quality factor $f_{\text {Rabi }} / \Gamma_{2}^{*}$. We leave such optimization for future work, hopefully aided by input from experiments. Nevertheless, we do emphasize one important feature that arises upon decreasing the tunnel coupling $V_{\mathrm{t}}$ with respect to the working point discussed above. Namely, spin-conserving uphill charge transitions from $\left|S_{b}\right\rangle$ to $\left|S_{a}\right\rangle$ can destabilize the qubit if the energy gap between the two electronic states is too narrow. The corresponding leakage rate can be calculated, e.g., from Eq. (16b) by using $\mathcal{T} \approx 1$ and $f_{\mathrm{L}} \mapsto \sqrt{2} V_{t} / h$. For example, at a relatively low tunnel coupling value $V_{t}=h \times 1.8 \mathrm{GHz}$, this leakage rate is $\approx 1 \mathrm{MHz}$, and is dominantly induced by $1 / f$ charge noise.

\section{CONCLUSIONS}

In conclusion, we consider information-loss mechanisms for an electrically controllable phosphorus nuclearspin qubit in a silicon nanostructure. We identify a parameter set (working point) where the information-loss time scales are longer than the estimated control time, suggesting that this setup is suited to demonstrate coherent electrical control of a nuclear spin. In this working point, the dominant decoherence mechanism is dephasing due to $1 / f$ charge noise. Our results are expected to facilitate the optimized design of nanostructures for quantum information experiments with nuclear-spin qubits.

\section{ACKNOWLEDGMENTS}

We thank W. A. Coish, L. Cywinski, P. HarveyCollard, V. Srinivasa for useful discussions. This research was supported by the National Research Development and Innovation Office of Hungary within the Quantum Technology National Excellence Program (Project No. 2017-1.2.1-NKP-2017-00001), and Grant 124723. A. P. was supported by the New National Excellence Program of the Ministry of Human Capacities.

\section{Appendix A: Estimate of the strength of the $1 / f$ charge noise}

In this appendix, we provide the details on how we estimated the strength $\alpha_{1 / f}$ of the $1 / f$ charge noise, as defined in Eq. (7).

Our first estimate is based on Ref. 14, which provides a realistic characterization of the power spectrum of
$1 / f$ electric-field fluctuation in silicon-based nanostructures similar to the one considered in the present work. Namely, at $T=100 \mathrm{mK}$, they use the electric-field noise spectrum

$$
S_{E}(f)=\frac{\beta_{E}}{2 \pi f},
$$

with $\beta_{E}=\frac{1}{6} 10^{4} \mathrm{~V}^{2} / \mathrm{m}^{2}$. This is converted to on-site energy fluctuation via $S_{\delta U}=e^{2} d^{2} S_{E}$, where $d$ is the distance between the charge center of the interface-bound charge state $|i\rangle$ and the charge center of the donor-bound charge state $|d\rangle$. Using this result for $S_{\delta U}$, Eq. (7) as a definition for $\alpha_{1 / f}$, and the parameter values $d=15 \mathrm{~nm}$ and $T=100 \mathrm{mK}$, we can express $\alpha_{1 / f} \approx 43.5 \mathrm{neV}$, as quoted in the main text below Eq. (7).

Our second estimate is based on the recent electron spin qubit experiment reported in Ref. 26. Their Fig. 4b shows the spectral density $S_{\delta f_{L}}$ of the fluctuations $\delta f_{L}$ of the spin-qubit Larmor frequency $f_{L}$. From that loglog plot, we can read off that the data is well described by the relation $y \approx 6.5-x$, where $y=\log _{10} S_{\delta f_{L}}$, and $x=\log _{10} f$. This is directly converted to

$$
S_{\delta f_{L}}(f) \approx \frac{3 \times 10^{6} \mathrm{~s}^{-2}}{f} \approx \frac{2 \times 10^{7} \mathrm{~s}^{-2}}{2 \pi f} \equiv \frac{\beta_{\delta f_{L}}}{2 \pi f} .
$$

Ref. 26 uses a simple model that establishes a linear relation between the qubit Larmor frequency fluctuation $\delta f_{L}$ and the quantum-dot on-site energy fluctuation $\delta U$, $\delta U=\gamma \delta f_{L}$. This implies that the on-site energy fluctuation is characterized by the spectral density

$$
S_{\delta U}(f)=\gamma^{2} S_{\delta f_{L}}(f)
$$

which, together with our Eqs. (7) and (A2), yields

$$
\alpha_{1 / f}=\frac{\gamma^{2} \beta_{\delta f_{L}}}{k_{B} T} .
$$

To obtain an estimate for our target quantity $\alpha_{1 / f}$, we need to express $\gamma$ first. To this end, we use the model of Ref. 26, which is based on the physical picture that the $1 / f$ noise is caused by fluctuating charge traps, located at a typical distance $d_{\text {ct }}$ from the center of the quantum dot. Within their model, the scaling factor $\gamma$ is given by

$$
\gamma=\frac{h m \omega_{0}^{2} d_{\mathrm{ct}}}{g \mu_{B} b_{\mathrm{long}}}
$$

Here, $m \approx 0.2 m_{\mathrm{e}}$ is the relevant conduction-band effective mass in silicon, $\hbar \omega_{0}$ is the orbital level spacing of the quantum dot, $g \approx 2$ is the effective electronic gfactor, and $b_{\text {long }}$ is the micromagnet-induced gradient of the longitudinal magnetic field. Using Eqs. (A2) and (A5) in Eq. (A4), and inserting the parameter values $\hbar \omega_{0}=1 \mathrm{meV}, b_{\text {long }}=0.2 \mathrm{mT} / \mathrm{nm}, d_{\mathrm{ct}}=100 \mathrm{~nm}$, and $T=100 \mathrm{mK}$, we obtain the value $\alpha_{1 / f} \approx 5.1 \mathrm{neV}$, as quoted in the main text below Eq. (7). 
* bence.hetenyi@unibas.ch

† palyi@mail.bme.hu

1 B. E. Kane, "A silicon-based nuclear spin quantum computer," Nature 393, 133 (1998).

2 Floris A. Zwanenburg, Andrew S. Dzurak, Andrea Morello, Michelle Simmons, Lloyd Hollenberg, Gerhard Klimeck, Sven Rogge, Susan Coppersmith, and Mark Eriksson, "Silicon quantum electronics," Rev. Mod. Phys. 85, 961 (2013).

3 M. Steger, K. Saeedi, M. L. W. Thewalt, J. J. L. Morton, H. Riemann, N. V. Abrosimov, P. Becker, and H.-J. Pohl, "Quantum Information Storage for over $180 \mathrm{~s}$ Using Donor Spins in a 28Si "Semiconductor Vacuum"," Science 336, 1280-1283 (2012).

4 Juha T. Muhonen, Juan P. Dehollain, Arne Laucht, Fay E. Hudson, Rachpon Kalra, Takeharu Sekiguchi, Kohei M. Itoh, David N. Jamieson, Jeffrey C. McCallum, Andrew S. Dzurak, and Andrea Morello, "Storing quantum information for 30 seconds in a nanoelectronic device," Nat Nano 9, 986-991 (2014).

${ }^{5}$ Kamyar Saeedi, Stephanie Simmons, Jeff Z. Salvail, Phillip Dluhy, Helge Riemann, Nikolai V. Abrosimov, Peter Becker, Hans-Joachim Pohl, John J. L. Morton, and Mike L. W. Thewalt, "Room-temperature quantum bit storage exceeding 39 minutes using ionized donors in silicon-28," Science 342, 830-833 (2013).

6 Jarryd J. Pla, Kuan Y. Tan, Juan P. Dehollain, Wee H. Lim, John J. L. Morton, Floris A. Zwanenburg, David N. Jamieson, Andrew S. Dzurak, and Andrea Morello, "Highfidelity readout and control of a nuclear spin qubit in silicon," Nature 496, 334-338 (2013).

7 Solomon Freer, Stephanie Simmons, Arne Laucht, Juha T Muhonen, Juan P Dehollain, Rachpon Kalra, Fahd A Mohiyaddin, Fay E Hudson, Kohei M Itoh, Jeffrey C McCallum, David N Jamieson, Andrew S Dzurak, and Andrea Morello, "A single-atom quantum memory in silicon," Quantum Science and Technology 2, 015009 (2017).

8 Juan P. Dehollain, Stephanie Simmons, Juha T. Muhonen, Rachpon Kalra, Arne Laucht, Fay Hudson, Kohei M. Itoh, David N. Jamieson, Jeffrey C. McCallum, Andrew S. Dzurak, and Andrea Morello, "Bell's inequality violation with spins in silicon," Nature Nanotechnology 11, 242 EP $-(2015)$.

9 J. T. Muhonen, J. P. Dehollain, A. Laucht, S. Simmons, R. Kalra, F. E. Hudson, A. S. Dzurak, A. Morello, D. N. Jamieson, J. C. McCallum, et al., "Coherent control via weak measurements in P 31 single-atom electron and nuclear spin qubits," Physical Review B 98, 155201 (2018).

10 Arne Laucht, Juha T. Muhonen, Fahd A. Mohiyaddin, Rachpon Kalra, Juan P. Dehollain, Solomon Freer, Fay E. Hudson, Menno Veldhorst, Rajib Rahman, Gerhard Klimeck, Kohei M. Itoh, David N. Jamieson, Jeffrey C. McCallum, Andrew S. Dzurak, and Andrea Morello, "Electrically controlling single-spin qubits in a continuous microwave field," Science Advances 1 (2015), 10.1126/sciadv.1500022.

11 Andrea Morello, Jarryd J. Pla, Floris A. Zwanenburg, Kok W. Chan, Kuan Y. Tan, Hans Huebl, Mikko Mottonen, Christopher D. Nugroho, Changyi Yang, Jessica A. van Donkelaar, Andrew D. C. Alves, David N. Jamieson, Christopher C. Escott, Lloyd C. L. Hollenberg, Robert G.
Clark, and Andrew S. Dzurak, "Single-shot readout of an electron spin in silicon," Nature 467, 687-691 (2010).

12 Jarryd J. Pla, Kuan Y. Tan, Juan P. Dehollain, Wee H. Lim, John J. L. Morton, David N. Jamieson, Andrew S. Dzurak, and Andrea Morello, "A single-atom electron spin qubit in silicon," Nature 489, 541-545 (2012).

13 Péter Boross, Gábor Széchenyi, and András Pályi, "Hyperfine-assisted fast electric control of dopant nuclear spins in semiconductors," Physical Review B 97, 245417 (2018).

14 Guilherme Tosi, Fahd A. Mohiyaddin, Vivien Schmitt, Stefanie Tenberg, Rajib Rahman, Gerhard Klimeck, and Andrea Morello, "Silicon quantum processor with robust longdistance qubit couplings," Nature Communications 8, 450 (2017).

15 Anthony J. Sigillito, Alexei M. Tyryshkin, Thomas Schenkel, Andrew A. Houck, and Stephen A. Lyon, "Allelectric control of donor nuclear spin qubits in silicon," Nature Nanotechnology 12, 958 (2017).

16 Stefan Thiele, Franck Balestro, Rafik Ballou, Svetlana Klyatskaya, Mario Ruben, and Wolfgang Wernsdorfer, "Electrically driven nuclear spin resonance in single-molecule magnets," Science 344, 1135-1138 (2014).

17 C. Godfrin, A. Ferhat, R. Ballou, S. Klyatskaya, M. Ruben, W. Wernsdorfer, and F. Balestro, "Operating Quantum States in Single Magnetic Molecules: Implementation of Grover's Quantum Algorithm," Phys. Rev. Lett. 119, 187702 (2017).

18 Guilherme Tosi, Fahd A Mohiyaddin, Stefanie Tenberg, Arne Laucht, and Andrea Morello, "Robust electric dipole transition at microwave frequencies for nuclear spin qubits in silicon," Physical Review B 98, 075313 (2018).

19 G. P. Lansbergen, R. Rahman, C. J. Wellard, I. Woo, J. Caro, N. Collaert, S. Biesemans, G. Klimeck, L. C. L. Hollenberg, and S. Rogge, "Gate-induced quantumconfinement transition of a single dopant atom in a silicon finfet," Nat. Phys. 4, 656 (2008).

20 Matias Urdampilleta, Anasua Chatterjee, Cheuk Chi Lo, Takashi Kobayashi, John Mansir, Sylvain Barraud, Andreas C. Betz, Sven Rogge, M. Fernando Gonzalez-Zalba, and John J. L. Morton, "Charge dynamics and spin blockade in a hybrid double quantum dot in silicon," Phys. Rev. X 5, 031024 (2015).

21 Patrick Harvey-Collard, N. Tobias Jacobson, Martin Rudolph, Jason Dominguez, Gregory A. Ten Eyck, Joel R. Wendt, Tammy Pluym, John King Gamble, Michael P. Lilly, Michel Pioro-Ladrière, and Malcolm S. Carroll, "Coherent coupling between a quantum dot and a donor in silicon," Nature Communications 8, 1029 (2017).

22 M. Rudolph, P. Harvey-Collard, R. Jock, T. Jacobson, J. Wendt, T. Pluym, J. Domnguez, G. Ten-Eyck, R. Manginell, M. P. Lilly, and M. S. Carroll, "Coupling mos quantum dot and phosphorous donor qubit systems," in 2016 IEEE International Electron Devices Meeting (IEDM) (2016) pp. 34.1.1-34.1.4.

23 Péter Boross, Gábor Széchenyi, and András Pályi, "Valley-enhanced fast relaxation of gate-controlled donor qubits in silicon," Nanotechnology 27, 314002 (2016).

24 Peihao Huang and Garnett W Bryant, "Spin relaxation of a donor electron coupled to interface states," Physical Review B 98, 195307 (2018). 
25 B. M. Freeman, J. S. Schoenfield, and H. W. Jiang, "Comparison of low frequency charge noise in identically patterned $\mathrm{Si} / \mathrm{SiO} 2$ and Si/SiGe quantum dots," Appl. Phys. Lett. 108, 253108 (2016).

${ }^{26}$ Jun Yoneda, Kenta Takeda, Tomohiro Otsuka, Takashi Nakajima, Matthieu R. Delbecq, Giles Allison, Takumu Honda, Tetsuo Kodera, Shunri Oda, Yusuke Hoshi, Noritaka Usami, Kohei M. Itoh, and Seigo Tarucha, "A quantum-dot spin qubit with coherence limited by charge noise and fidelity higher than 99.9\%," Nature Nanotechnology 13, 102-106 (2018).

27 Yuriy Makhlin, Gerd Schön, and Alexander Shnirman, "Dissipation in josephson qubits," in New Directions in Mesoscopic Physics (Towards Nanoscience) (Springer, 2003) pp. 197-224.

${ }^{28}$ Sh. Kogan, Electronic Noise and Fluctuations in Solids (Cambridge University Press, New York, 1996).

${ }^{29}$ L. Petit, J. M. Boter, H. G. J. Eenink, G. Droulers, M. L. V. Tagliaferri, R. Li, D. P. Franke, K. J. Singh, J. S. Clarke, R. N. Schouten, V. V. Dobrovitski, L. M. K. Vandersypen, and M. Veldhorst, "Spin lifetime and charge noise in hot silicon quantum dot qubits," Phys. Rev. Lett. 121, 076801 (2018).

30 S. W. Jung, T. Fujisawa, Y. Hirayama, and Y. H. Jeong, "Background charge fluctuation in a GaAs quantum dot device," Applied Physics Letters 85, 768-770 (2004).

31 C. Dekker, A. J. Scholten, F. Liefrink, R. Eppenga, H. van Houten, and C. T. Foxon, "Spontaneous resistance switching and low-frequency noise in quantum point contacts," Phys. Rev. Lett. 66, 2148-2151 (1991).

32 Patrick Harvey-Collard, Benjamin DAnjou, Martin Rudolph, N Tobias Jacobson, Jason Dominguez, Gregory A Ten Eyck, Joel R Wendt, Tammy Pluym, Michael P Lilly, William A Coish, et al., "High-fidelity single-shot readout for a spin qubit via an enhanced latching mecha- nism," Physical Review X 8, 021046 (2018).

33 Roland Winkler, Spin-orbit Coupling Effects in TwoDimensional Electron and Hole Systems, Springer Tracts in Modern Physics, Vol. 191 (Springer, 2003).

34 Conyers Herring and Erich Vogt, "Transport and deformation-potential theory for many-valley semiconductors with anisotropic scattering," Phys. Rev. 101, 944-961 (1956).

35 Peter Y. Yu and Manuel Cardona, Fundamentals of Semiconductors (Springer, Berlin, 2010).

36 A. A. Clerk, M. H. Devoret, S. M. Girvin, Florian Marquardt, and R. J. Schoelkopf, "Introduction to quantum noise, measurement, and amplification," Rev. Mod. Phys. 82, 1155-1208 (2010).

37 M. Veldhorst, R. Ruskov, C. H. Yang, J. C. C. Hwang, F. E. Hudson, M. E. Flatté, C. Tahan, K. M. Itoh, A. Morello, and A. S. Dzurak, "Spin-orbit coupling and operation of multivalley spin qubits," Phys. Rev. B 92, 201401 (2015).

38 Ryan M. Jock, N. Tobias Jacobson, Patrick HarveyCollard, Andrew M. Mounce, Vanita Srinivasa, Dan R. Ward, John Anderson, Ron Manginell, Joel R. Wendt, Martin Rudolph, Tammy Pluym, John King Gamble, Andrew D. Baczewski, Wayne M. Witzel, and Malcolm S. Carroll, "A silicon metal-oxide-semiconductor electron spin-orbit qubit," Nature Communications 9, 1768 (2018).

39 P. Tanttu et al., "Controlling spin-orbit interactions in silicon quantum dots using magnetic field direction," (), arXiv:1807.10415 (unpublished).

40 P. Harvey-Collard et al., "Implications of the spin-orbit interaction for singlet-triplet qubits in silicon," (), arXiv:1808.07378 (unpublished).

41 Alexander Shnirman, Yuriy Makhlin, and Gerd Schön, "Noise and decoherence in quantum two-level systems," Physica Scripta 2002, 147 (2002). 